\title{
Multipartite Einstein-Podolsky-Rosen steering and genuine tripartite entanglement with optical networks
}

\author{
Seiji Armstrong ${ }^{1 \star}$, Meng Wang ${ }^{2,3}$, Run Yan Teh ${ }^{4}$, Qihuang Gong ${ }^{2,3}$, Qiongyi He ${ }^{2,3,4 \star}$, Jiri Janousek ${ }^{1}$, \\ Hans-Albert Bachor ${ }^{1}$, Margaret D. Reid ${ }^{4 \star}$ and Ping Koy Lam ${ }^{1,5}$
}

Einstein, Podolsky and Rosen (EPR) pointed out in their famous paradox that two quantum-entangled particles can have perfectly correlated positions and momenta'. Such correlations give evidence for the nonlocality of quantum mechanics ${ }^{2}$ and form the basis for quantum cryptography ${ }^{3}$ and teleportation $^{4}$. EPR steering ${ }^{5-8}$ is the nonlocality associated with the EPR paradox and has traditionally been investigated between only two parties ${ }^{9-14}$. Using optical networks and efficient detection, we present experimental observations of multiparty EPR steering and of the genuine entanglement of three intense optical beams. We entangle the quadrature phase amplitudes of distinct fields, in analogy to the position-momentum entanglement of the original paradox. Our experiments complement tests of quantum mechanics that have entangled small systems ${ }^{15-17}$ or have demonstrated tripartite inseparability $^{18,19}$. Our methods establish principles for the development of multiparty quantum communication protocols with asymmetric observers, and can be extended to qubits, whether photonic $^{11-13,15,17}$, atomic $^{16}$, superconducting ${ }^{20}$, or otherwise.

Schrödinger introduced the term 'steering' to describe the 'spooky action-at-a-distance' nonlocality apparent in the EPR paradox ${ }^{1}$, and pointed out that these states involve a quantum property called 'entanglement ${ }^{5,21}$. Wiseman et al. ${ }^{6,7}$ have formalized the meaning of steering in terms of violations of local hiddenstate models, and revealed that the EPR paradox is a manifestation of steering. The name EPR steering has been coined to describe this effect ${ }^{8}$, not given by all entangled states, where measurements made by one observer can apparently 'steer' (alter) the state of another observer at a different location. EPR steering offers insights into directional nonlocality ${ }^{22}$ and differs conceptually from entanglement, which is defined symmetrically with respect to both observers ${ }^{21}$.

The motivation to observe multipartite EPR steering and entanglement arises from the need to understand whether and how this type of entanglement can be distributed over many different systems. We expand on the theoretical framework of refs 17,23 and derive and use criteria to detect the multipartite EPR steering and genuine tripartite entanglement between macroscopic optical systems.

Further, motivated by considerations of real-world quantum networks such as the quantum internet ${ }^{24}$, for which security and privacy are of paramount importance, we investigate properties of multipartite steering relevant to quantum communication. We demonstrate relations that give bounds on how steering can be distributed among different parties, linking the amount of steering to the security of channels in the network. We also demonstrate the principle of 'one-sided device-independent' quantum secret sharing, a protocol used to protect a highly important message, by ensuring that two (potentially untrustworthy) parties must collaborate to gain access to information held by a third party $y^{25}$. The collaborating parties need not assume reliable devices ${ }^{26}$. Last, we study the effect of losses on the steering in the network. For two-party entangled states, it is possible to block the steering in a given direction, to create three regimes: two-way, one-way and no-way steering 22 . By introducing asymmetric losses, we reveal that similar directional steering and manipulation is possible for larger multipartite states. We demonstrate this on a seven-quantum mode (qumode) state.

Underpinning the idea of multipartite EPR steering is the quantum concept of entanglement ${ }^{21}$. Two systems are entangled if their overall quantum state cannot be expressed as a product of two quantum states, one for each system (or as a mixture of such products). $N$ systems are genuinely $N$-partite entangled if (and only if) the entanglement of the $N$-party system cannot be produced by mixing quantum states with fewer than $N$ systems entangled ${ }^{17}$. Suppose three observers (Alice, Bob and Charlie) each make measurements on three respective quantum systems, labelled 1,2, and 3. We show in the Supplementary Information that genuine tripartite entanglement of the three systems can be confirmed if:

$$
\Delta\left(\hat{x}_{1}-\frac{\left(\hat{x}_{2}+\hat{x}_{3}\right)}{\sqrt{2}}\right) \times \Delta\left(\hat{p}_{1}+\frac{\left(\hat{p}_{2}+\hat{p}_{3}\right)}{\sqrt{2}}\right)<1
$$

Here $\hat{x}_{i}$ and $\hat{p}_{i}(i=1,2,3)$ are the position and momentum observables of the system $i$, scaled in such a way that the Heisenberg uncertainty relation becomes $\Delta \hat{x}_{i} \Delta \hat{p}_{i} \geq 1$. We define $(\Delta \hat{x})^{2}=\left\langle\hat{x}^{2}\right\rangle-\langle\hat{x}\rangle^{2}$. Genuine tripartite entanglement has a different meaning to full tripartite inseparability ${ }^{17}$. The latter occurs when the entanglement cannot be produced by entangling any (fixed) two parties, and does not eliminate that entanglement is created by mixing different bipartite entangled states. Criteria for genuine

${ }^{1}$ Centre for Quantum Computation and Communication Technology, Department of Quantum Science, The Australian National University, Canberra, ACT 0200, Australia. ${ }^{2}$ State Key Laboratory for Mesoscopic Physics, School of Physics, Peking University, Beijing 100871, China. ${ }^{3}$ Collaborative Innovation Center of Quantum Matter, Beijing 100871, China. ${ }^{4}$ Centre for Quantum and Optical Science, Swinburne University of Technology, Melbourne, Victoria 3122, Australia. ${ }^{5}$ College of Precision Instrument and Opto-Electronics Engineering, Tianjin University, Key Laboratory of Opto-Electronics Information Technology, Ministry of Education, Tianjin, 300072, China. *e-mail: seiji.armstrong@gmail.com; qiongyihe@pku.edu.cn; mdreid@swin.edu.au 


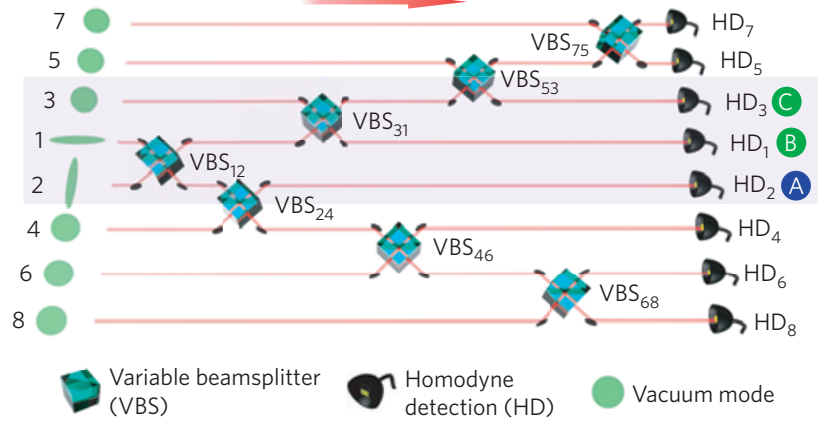

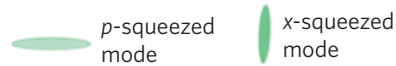

Figure 1 | Programmable linear optics circuit for demonstrating multipartite steering. Independent qumodes are shaped to be multiplexed on the same beam. By programmatically changing the measurement basis, the scheme allows us to emulate linear optics networks in real time. The circuit employs two quadrature-squeezed qumodes and six quantum-noise limited vacuum modes as inputs. Each of the seven beamsplitters are fully tunable from $R=0 \%$ to $R=100 \%$. We set a beamsplitter reflectivity to $R=100 \%$ and set it to a perfect mirror when we choose not to mix a particular input qumode into the state. Optimal circuits are created that take into account asymmetries in squeezing values of the input qumodes.

tripartite entanglement using position and momentum have been derived previously ${ }^{17}$, but for the states we generate in our experiment criterion (1) becomes necessary.

To understand tripartite EPR steering, we again consider that Alice's measurements are the observables $\hat{x}_{1}$ and $\hat{p}_{1}$ of a quantum system. However, this assumption no longer applies to Bob and Charlie, who need not report the results of quantum observables. We suppose that Bob and Charlie can collaborate to give a prediction for the outcome of Alice's $\hat{x}_{1}$ (or $\hat{p}_{1}$ ) measurement and denote the average uncertainty in their inferences by $\Delta_{\text {inf }}\left(\hat{x}_{1}\right)$ (and $\Delta_{\text {inf }}\left(\hat{p}_{1}\right)$ ). If $S_{1}=\Delta_{\text {inf }}\left(\hat{x}_{1}\right) \Delta_{\text {inf }}\left(\hat{p}_{1}\right)<1$, then we realize an EPR paradox ${ }^{27}$. In that case, the EPR assumption that the actions of Bob and Charlie cannot immediately affect Alice's system suggests that Alice's $\hat{x}_{1}$ and $\hat{p}_{1}$ values are predetermined to an accuracy contradicting quantum mechanics ${ }^{1}$. The condition gives the inconsistency of quantum mechanics with the EPR premises, and thus the EPR paradox. It is also the condition for which there is a 'steering' of Alice's system by Bob and Charlie's actions ${ }^{5}$. We symbolize this directional EPR steering by the notation $\mathrm{BC} \rightarrow \mathrm{A}$, where $\mathrm{A}, \mathrm{B}$ and $\mathrm{C}$ are the sites of Alice, Bob and Charlie.

Next, we extend to $N$ observers. Any $N$-party state demonstrates $N$-partite EPR steering of the $j$ th site by the remaining set of sites denoted $K$ if

$$
S_{j \mid K} \equiv \Delta_{\text {inf }}\left(\hat{x}_{j}\right) \Delta_{\text {inf }}\left(\hat{p}_{j}\right)<1
$$

We define the square of this product, $\left(S_{j \mid K}\right)^{2}$, to be the EPR steering number. In our experiment, the steering measurements are optimized linear combinations of the $\hat{x}_{k}\left(\hat{p}_{k}\right)(k \neq j)$. Thus

$$
\begin{aligned}
& \Delta_{\text {inf }}\left(\hat{x}_{j}\right)=\Delta\left(\hat{x}_{j}+\sum_{k \neq j} g_{k, x} x_{k}\right) \\
& \Delta_{\text {inf }}\left(\hat{p}_{j}\right)=\Delta\left(\hat{p}_{j}+\sum_{k \neq j} g_{k, p} p_{k}\right)
\end{aligned}
$$

where $g_{x, k}$ and $g_{p, k}$ are optimized real numbers. The steering detected by equation (2) is genuinely $N$-partite if it cannot be explained as arising from any steering limited to $N-1$ or fewer parties.
Table 1 | Beamsplitter reflectivities (\%) for optimized optics networks.

\begin{tabular}{llllllll}
$\mathbf{N}$ & $\mathbf{V B S}_{\mathbf{1 2}}$ & $\mathbf{V B S}_{\mathbf{3 1}}$ & $\mathbf{V B S}_{\mathbf{2 4}}$ & $\mathbf{V B S}_{\mathbf{5 3}}$ & $\mathbf{V B S}_{\mathbf{4 6}}$ & $\mathbf{V B S}_{\mathbf{7 5}}$ & $\mathbf{V B S}_{\mathbf{6 8}}$ \\
\hline 2 & 50 & 100 & 100 & 100 & 100 & 100 & 100 \\
3 & 51.1 & 50 & 100 & 100 & 100 & 100 & 100 \\
4 & 50 & 50 & 50 & 100 & 100 & 100 & 100 \\
5 & 50.8 & 33.3 & 50 & 50 & 100 & 100 & 100 \\
6 & 50 & 33.3 & 33.3 & 50 & 50 & 100 & 100 \\
7 & 50.6 & 25 & 33.3 & 33.3 & 50 & 50 & 100 \\
8 & 50 & 25 & 25 & 33.3 & 33.3 & 50 & 50
\end{tabular}

In the tripartite case, all fixed two-party steering is negated, if we can demonstrate each of ${ }^{23}$

$$
S_{\mathrm{A} \mid \mathrm{BC}}<1, S_{\mathrm{B} \mid \mathrm{AC}}<1, S_{\mathrm{C} \mid \mathrm{AB}}<1
$$

which implies steering across all bipartitions: $\mathrm{BC} \rightarrow \mathrm{A}, \mathrm{AC} \rightarrow \mathrm{B}$ and $\mathrm{AB} \rightarrow \mathrm{C}$. This condition tells us that the position and momentum amplitudes of any one of the parties is highly correlated with a combination of amplitudes of the other two parties. The correlation can be used to establish a sequence of numbers (a quantum key) that is shared between the two groups for the encryption and decryption of messages between them. This quantum key distribution can be established in a one-sided device-independent way, without the assumption of trust of devices on any but the single party. The condition (3) confirms the full inseparability of the quantum density matrix and also of any three-party hidden-state model that could describe the system ${ }^{6}$.

To claim genuine tripartite steering we need to rule out that the steering has been created by mixing states with two-party steering across different bipartitions $s^{23}$. We prove in the Supplementary Information that this is done if

$$
\Delta\left(\hat{x}_{1}-\frac{\left(\hat{x}_{2}+\hat{x}_{3}\right)}{\sqrt{2}}\right) \times \Delta\left(\hat{p}_{1}+\frac{\left(\hat{p}_{2}+\hat{p}_{3}\right)}{\sqrt{2}}\right)<0.5
$$

which is a stricter form of inequality equation (1). Our present experiment meets the conditions (1) and (3).

We investigate multipartite entanglement and steering by employing the programmable linear optics circuit developed in ref. 19 to create various multipartite quantum states from different networks. The various quantum networks that we create for this demonstration can be visualized in the programmable circuit of Fig. 1.

Homodyne detection is employed to measure the inferred variances of each qumode in the state. This provides highly efficient detection, and our measurements of the amplitudes do not rely on fair sampling assumptions ${ }^{12,13}$. Each qumode is characterized by the conjugate quadrature operators $\hat{x}$ and $\hat{p}$ of the quantum harmonic oscillator mode of the light field.

In all of our experimental demonstrations we input two mixed states with different magnitudes of squeezing, and six quantumnoise limited vacuum modes, allowing us to explore how best to bias and optimize the beamsplitter networks,

$$
\left(\begin{array}{c}
\left(\Delta \hat{p}_{1}\right)^{2} \\
\left(\Delta \hat{x}_{1}\right)^{2} \\
\left(\Delta \hat{p}_{2}\right)^{2} \\
\left(\Delta \hat{x}_{2}\right)^{2}
\end{array}\right)=\left(\begin{array}{l}
-3.6 \pm 0.05 \mathrm{~dB} \\
+8.9 \pm 0.05 \mathrm{~dB} \\
+9.5 \pm 0.05 \mathrm{~dB} \\
-4.1 \pm 0.05 \mathrm{~dB}
\end{array}\right)
$$

The optimized linear optics networks that we create for our mixed inputs are detailed in the Methods. We first generate seven different quantum states from seven different networks, with each beamsplitter's reflectivity given in Table 1. 
a
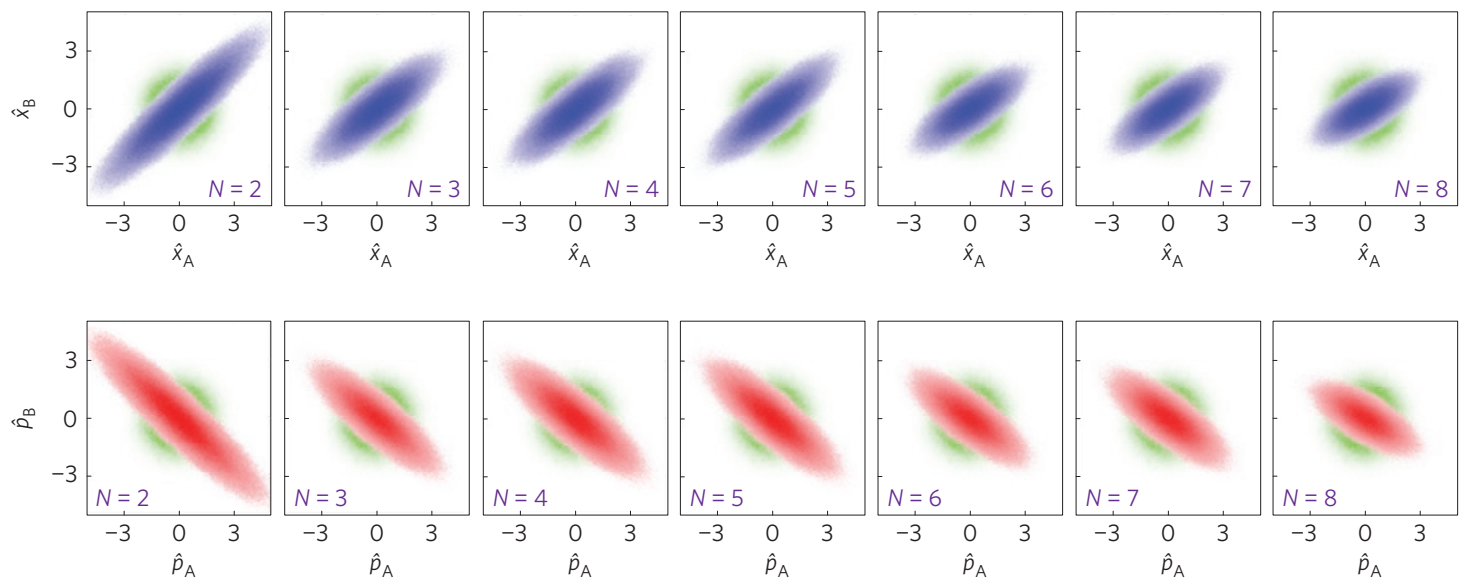

b

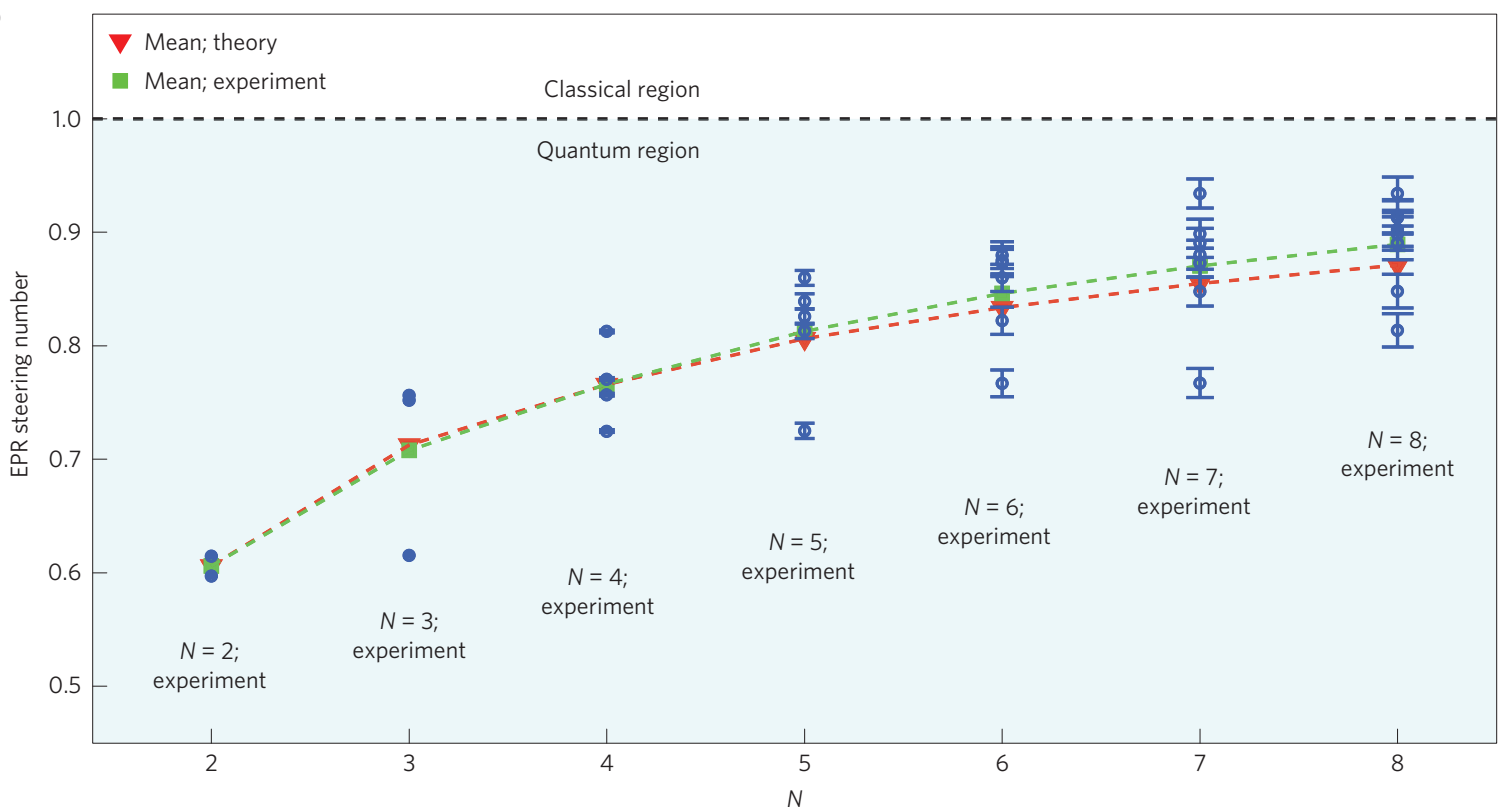

Figure 2 | Multipartite EPR Steering for the $\mathbf{N}$-partite entangled states, $\mathbf{N}=\mathbf{2 - 8}$. Here 'classical' means that this condition cannot be obtained for any optimized choice of gains $g_{k, x}$ and $g_{k, p}$. $\mathbf{a}$, Normalized quadrature amplitudes in $\hat{x}(\hat{p})$ are plotted parametrically to visualize correlations quantified by equation (2) in the main text. For all seven states, the $\hat{x}(\hat{p})$ quadrature amplitude of the first qumode in the $N$-qumode state is plotted against the linear combination of the $\hat{x}(\hat{p})$ quadrature amplitudes of the remaining qumodes. Green data points show shot-noise measurements, blue (red) data points show $\hat{x}(\hat{p})$ quadrature measurements. Correlations weaken as $N$ increases. $\mathbf{b}$, The blue markers give for each $N$-partite state the $N$ experimental values for the EPR steering number $\left(S_{j \mid K}\right)^{2}$, where we consider the steering of the jth qumode $(j=1, \ldots, N)$ by the remaining group $K$. The condition $\left(S_{j \mid K}\right)^{2}<1$ of inequality (2) for EPR steering of qumode $j$ is satisfied in each case. The experimental values are consistent with the theoretical predictions (red markers) explained in the Methods and Supplementary Section III D. The error bars are calculated from characterizations of imperfect homodyne detections of the spatial mode bases (Methods).

The EPR correlations in each state can be visualized by parametrically plotting components of the inferred variance terms. The ellipticity of each ellipse in Fig. $2 a$ is indicative of finite squeezing. We see that the higher the number of qumodes in the state, the weaker the correlations become. This is evident in the slight rotation off the strictly diagonal axis in both quadratures, as well as the diminishing ellipticity of the correlations. This is due to the additional vacuum contributions in our circuit as we go to higher mode numbers.

Figure $2 \mathrm{~b}$ quantifies the correlations in terms of the EPR steering number, equation (2). For each $N$-qumode state (up to $N=8$ ) there are $N$ EPR steering numbers that must be tested to confirm the multipartite EPR steering of each qumode by the remaining group of qumodes. Each EPR steering number represents a direction of steering within the state and we confirm multipartite steering in all cases (see also Supplementary Section III D). This implies low inference uncertainties for the amplitudes of each single qumode, by all the other parties, which is a requirement for a one-sided deviceindependent quantum key distribution between the two groups (trust required only for the single qumode). For $N=3$, we confirm steering across all bipartitions of the state, to satisfy criterion (3), thus ruling out a large class of separable classical models.

We next demonstrate properties of multipartite EPR steering which have implications for security in quantum communications. First, the manifestation of steering by inequality (2) is monogamous (Fig. 3a). This means that if such steering is shared between two parties, Alice and Bob, it cannot be shared between Alice and a third party, Charlie. Mathematically, the monogamy is described by $S_{\mathrm{A} \mid \mathrm{B}} S_{\mathrm{A} \mid \mathrm{C}} \geq 1$ (ref. 23). The EPR steering number $\left(S_{\mathrm{A} \mid \mathrm{B}}\right)^{2}$ therefore indicates the directional security of the A-B channel, as it gives a lower bound on the inferred uncertainty $S_{\mathrm{A} \mid \mathrm{C}}$ of Alice's amplitudes for any eavesdropping parties, 'Eve', also represented by C. Another 
a

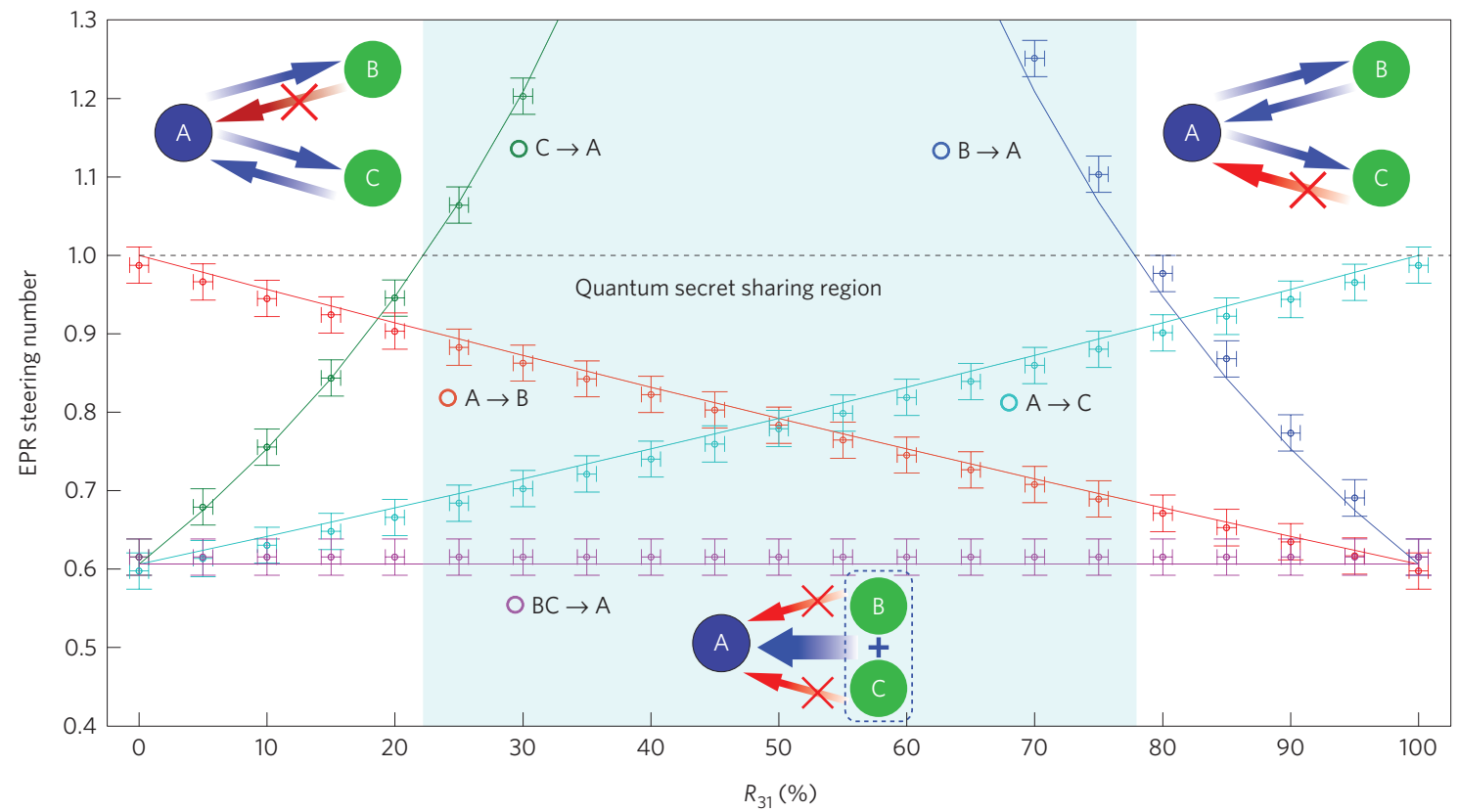

b

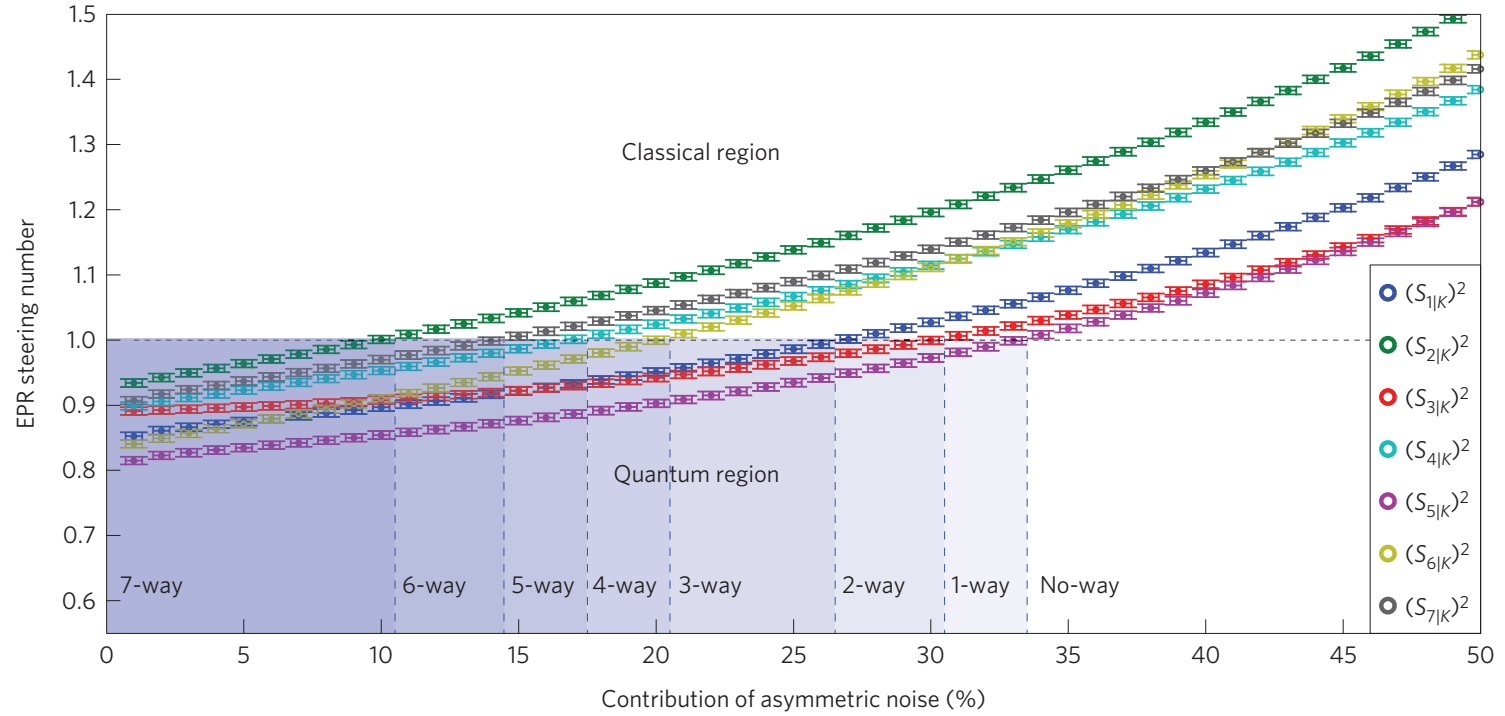

Figure 3 | Directional properties of multipartite steerable states. a, EPR steering numbers for $N=3$ versus the reflectivity $R_{31}$ of the second beamsplitter $\mathrm{VBS}_{31}$. The first beamsplitter, $\mathrm{VBS}_{12}$, is held constant at $R_{12}=51.1 \%$, and $R_{24}=R_{53}=100 \%$. Markers indicate experimental data, curves are theoretical predictions. The single qumode on one side of the first beamsplitter is denoted by $A$; the two qumodes on the other side by $B$ and $C$. Directional monogamy, where two parties (B and C) cannot simultaneously independently steer the same third system (A), is shown by the blue and red arrows in the cartoons. Thus, steering of $A$ by $B$ (or $C$ ) is possible only for asymmetric beamsplitters (blue and green markers). We confirm $S_{A \mid B} S_{A \mid C} \geq 1$. However, the simultaneous steering of $\mathrm{B}$ and $\mathrm{C}$ by $\mathrm{A}$ occurs for all $R_{31}$ (red and cyan markers). One-sided device-independent quantum secret sharing is shown in the blue regime of more symmetric beamsplitters, where neither Bob nor Charlie can independently steer A (red arrows), but they can steer A by collaboration (magenta markers). The error bars arise from imperfect homodyne detection of the three-qumode basis, see Methods. $\mathbf{b}$, Manipulating the directional steering of a seven-partite steerable state by introducing asymmetric noise. Eight different regimes are demonstrated. Details are given in the Supplementary Information. The error bars arise from imperfect homodyne detection of the seven-qumode basis, see Methods.

property of multipartite EPR steering is that, for some regimes, the steering of A cannot take place by Bob (or Charlie) alone, but requires both parties. This is relevant to secret sharing protocols. In Fig. 3a we illustrate the principle of one-sided device-independent quantum secret sharing ${ }^{25}$. The values of Alice's amplitudes can form the basis for a key, but can only be unlocked by Bob and Charlie with a low uncertainty if they combine measurement outcomes. As we do not assume quantum measurements for the steering parties, the security is independent of the exact nature of Bob or Eve/Charlie's measurement devices (although Alice's measurement station must be trusted-details are given in the Supplementary Information $)^{26}$.
To investigate losses, we modified the seven-qumode network to allow the systematic introduction of asymmetric noise into the network. This is done by adding variable loss to one half of the network via a vacuum coupling beamsplitter acting on one output arm of $\mathrm{VBS}_{12}$. The monogamy relation explains the impossibility of steering $S_{\mathrm{A} \mid \mathrm{B}}<1$ when the losses on the steering channel are $50 \%$ or greater ${ }^{28}$. Thus, one can manipulate the asymmetries to successively disable the steering of each one of the parties (Fig. 3b). In this way, we find that all eight separate steering regimes are accessible by introducing up to $33 \%$ of asymmetric loss into the circuit. The imperfect mode matching (over 98\% efficiencies) and imperfect 


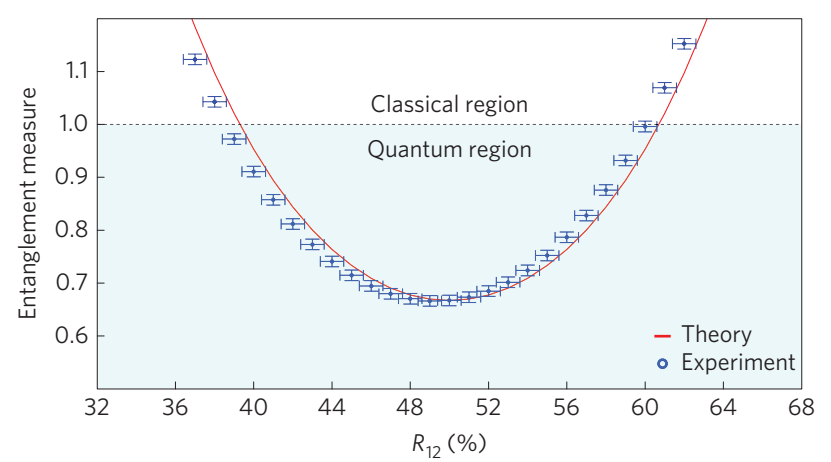

Figure 4 | Genuine tripartite entanglement. The values of the genuine tripartite entanglement parameter $\Delta^{2}\left(\hat{x}_{1}-\left(\hat{x}_{2}+\hat{x}_{3}\right) / \sqrt{2}\right)$ $\Delta^{2}\left(\hat{p}_{1}+\left(\hat{p}_{2}+\hat{p}_{3}\right) / \sqrt{2}\right)$ as given by equation (1) for the tripartite steerable state studied in Fig. 3. A balanced beamsplitter is employed for the second beamsplitter in the network. The experimental data (blue circles) shows agreement with the predictions of the theoretical model (red curve) outlined in the Methods. The data satisfies the inequality (1) and therefore confirms the genuine tripartite entanglement of three optical modes. We note that, for $R_{1} \neq 50 \%$, the genuine tripartite entanglement can be more sensitively detected using a criterion involving an asymmetric choice of amplitude weightings (see Supplementary Information). The error bars arise from imperfect homodyne detection. The systematic offset relative to predictions is probably due to a slight misalignment of the multiphotodiode homodyne detection scheme (Methods).

detection of each qumode together with the systematic loss allows for the separate regimes.

Finally, the beamsplitters were varied to switch from multipartite steering to genuine tripartite entanglement. In Fig. 4, we confirm equation (1) and hence the genuine tripartite entanglement of three qumodes. Although this sort of entanglement has been realized for three spatially separated photons ${ }^{15,17}$, our results demonstrate genuine tripartite entanglement in a very different scenario: namely, for the EPR observables of three fields consisting of many photons detected at very high efficiencies. As discussed in refs 12-14, considerations of loss become important for conclusive demonstrations of EPR steering, which is a form of quantum nonlocality. We do not reach the condition of equation (4), to confirm fully genuine tripartite steering, but our theoretical model predicts this to be possible, once the squeezing of inputs is improved to $\sim-6 \mathrm{~dB}$.

The methods developed here may motivate investigations of the nonlocality between asymmetric systems. One example is the entanglement of mechanical motion with the amplitudes of the radiation field in opto-mechanical systems ${ }^{29}$.

\section{Methods \\ Relationship between steering and entanglement. In the tripartite case, confirming steering of A by the group $\mathrm{BC}$ amounts to confirming entanglement between the two groups, but with fewer assumptions made about group BC. Steering is thus a one-sided device-independent way to verify entanglement $t^{6,7,26,30}$. This means we do not need to trust, for example, that Bob and Charlie calibrate their results reliably according to a given quantum measurement procedure, or even that the computers from which they report data have not been tampered with $^{30}$. It has been shown experimentally that the condition $S_{1}<1$ for EPR steering is not reached by all entangled state ${ }^{28}$. Steering thus represents a greater experimental challenge than entanglement, but once confirmed gives the advantage of validating quantum communication protocols based on entanglement, without the need for full trust of all the observers of a network.}

Parametric plots of multipartite states. In all plots of Fig. 2a, the $x$ axis is the $\hat{x}(\hat{p})$ quadrature amplitude of the first qumode in the state, and the $y$ axis is the linear combination of the $\hat{x}(\hat{p})$ quadrature amplitudes of the remaining $N-1$ qumodes in the $\mathrm{N}$-qumode state. Each distribution is represented as an intensity map of 1,000,000 data points binned in 122,500 bins. Perfect correlations would correspond to a semi-major axis of infinite length, strictly on the diagonal. This would require infinite energy and is unphysical. The trend of decaying EPR steering strength with higher $N$ evident in Fig. 2 can be mitigated by inputting $N$ squeezed state inputs (and no vacuum).

Optimizing asymmetric linear optics networks. The linear optics networks used to generate the results presented in Fig. 2a,b are characterized by the beamsplitters tabulated in Table 1 .

The familiar bipartite EPR state is generated by setting the first beamsplitter reflectivity to $R=50 \%$ and all other beamsplitters to mirrors. The measurement returns an EPR state and six unmixed vacuum modes, which are discarded. To create an $N$-qumode state we set $N-1$ of these to function as beamsplitters and the remaining $8-N$ to function as mirrors. We emphasize that although our qumodes are in principle spatially distinguishable, measurement events are not spacelike separated, and our work cannot address locality loopholes ${ }^{13}$. However, as noted in ref. 12, for practical quantum communication the issue of locality is often less problematic.

In all of our networks, when the two squeezed qumodes are pure states and equal in squeezing magnitude, the optimal beamsplitter reflectivity of the first beamsplitter is $R=50 \%$ (even for odd qumode numbers). For two mixed states that are unequal in squeezing values, the optimal beamsplitter ratio for even-numbered states remains $R=50 \%$. The symmetry arises from the EPR steering criteria being directional. The symmetry breaks down for mixed-state inputs when we consider odd-numbered quantum states. In this situation we benefit from biasing the beamsplitters away from $R=50 \%$.

It is only necessary to optimize the first beamsplitter of the network in odd-numbered quantum states. We see that the reflectivities are $51.1 \%, 50.8 \%$ and $50.6 \%$ for the three-qumode, five-qumode and seven-qumode states, respectively. For any asymmetry in the inputs this will converge to $50 \%$ for large $N$.

We calculate the moments corresponding to the criteria above, using the unitary transformation:

$a_{\text {out }, 1}=\sqrt{R} a_{\mathrm{in}, 1}+\sqrt{(1-R)} a_{\mathrm{in}, 2}, a_{\mathrm{out}, 2}=\sqrt{(1-R} a_{\mathrm{in}, 1}-\sqrt{R} a_{\mathrm{in}, 2}$ to model the interaction of the qumodes at the beamsplitter ${ }^{18}$. Here, $a_{\mathrm{out}, 1}$ and $a_{\mathrm{out}, 2}$ are the two output qumodes and $a_{\mathrm{in}, 1}$ and $a_{\mathrm{in}, 2}$ are the two qumodes input to the beamsplitter. The optimization gain parameters are tabulated in the Supplementary Information.

Squeezed light sources. We use two near-identical optical parametric amplifiers each containing a periodically poled potassium titanyl phosphate crystal. The generated squeezed light has a squeezed noise suppression of $-6.8 \pm 0.2 \mathrm{~dB}$ and an antisqueezed noise level of $8.7 \pm 0.2 \mathrm{~dB}$. The squeezers are pumped by $532 \mathrm{~nm}$ light from a frequency-doubled continuous-wave Nd:YAG laser, with the fundamental wavelength at $1,064 \mathrm{~nm}$.

Uncertainties arising from imperfect detection. The mode overlap of each qumode with the homodyne detection local oscillator is between $99.2 \%$ and $99.4 \%$. This leads to a minimum mode matching efficiency of $98.4 \%$. We use a Hamamatsu InGaAs PIN photodiode array (G7150) with a quantum efficiency of $80 \%$. The misalignment of the signal beam during detection due to thermal drifts and mechanical relaxations of optical mounts contribute to variances in the measured qumodes ranging from $0.02 \mathrm{~dB}$ up to $0.20 \mathrm{~dB}$. As larger networks use more vacuum modes, the uncertainties increase with higher $N$. The systematic discrepancy between the theory and experimental data in Fig. 4 can be explained by considering a slight experimental misalignment of the signal beam on the multiphotodiode homodyne detection scheme, resulting in a $0.5 \%$ offset in beamsplitter reflectivity.

Received 8 April 2014; accepted 20 November 2014; published online 5 January 2015

\section{References}

1. Einstein, A., Podolsky, B. \& Rosen, N. Can quantum-mechanical description of physical reality be considered complete? Phys. Rev. 47, 777-780 (1935).

2. Bell, J. S. On the Einstein Podolsky Rosen paradox. Physics 1, 195-200 (1964).

3. Scarani, V. et al. The security of practical quantum key distribution. Rev. Mod. Phys. 81, 1301-1350 (2009).

4. Furusawa, A. et al. Unconditional quantum teleportation. Science 23, 706-709 (1998).

5. Schrödinger, E. Discussion of probability relations between separated systems. Proc. Camb. Phil. Soc. 31, 555-563 (1935).

6. Wiseman, H. M., Jones, S. J. \& Doherty, A. C. Steering, entanglement, nonlocality, and the Einstein-Podolsky-Rosen paradox. Phys. Rev. Lett. 98, 140402 (2007).

7. Jones, S. J., Wiseman, H. M. \& Doherty, A. C. Entanglement, Einstein-Podolsky-Rosen correlations, Bell nonlocality, and steering. Phys. Rev. A 76, 052116 (2007). 
8. Cavalcanti, E. G., Jones, S. J., Wiseman, H. M. \& Reid, M. D. Experimental criteria for steering and the Einstein-Podolsky-Rosen paradox. Phys. Rev. A 80, 032112 (2009).

9. Ou, Z. Y., Pereira, S. F., Kimble, H. J. \& Peng, K. C. Realization of the Einstein-Podolsky-Rosen paradox for continuous variables. Phys. Rev. Lett. 68, 3663-3666 (1992).

10. Reid, M. D. et al. Colloquium: The Einstein-Podolsky-Rosen paradox: From concepts to applications. Rev. Mod. Phys. 81, 1727-1751 (2009).

11. Saunders, D., Jones, S., Wiseman, H. M. \& Pryde, G. J. Experimental EPR-steering using Bell-local states. Nature Phys. 6, 845-849 (2010).

12. Smith, D. H. et al. Conclusive quantum steering with superconducting transition-edge sensors. Nature Commun. 3, 625 (2012).

13. Wittmann, B. et al. A. Loophole-free Einstein-Podolsky-Rosen experiment via quantum steering. New J. Phys. 14, 053030 (2012).

14. Bennet, A. J. et al. Arbitrarily loss-tolerant Einstein-Podolsky-Rosen steering allowing a demonstration over $1 \mathrm{~km}$ of optical fiber with no detection loophole. Phys. Rev. X 2, 031003 (2012).

15. Lavoie, J., Kaltenbaek, R. \& Resch, K. J. Experimental violation of Svetlichny's inequality. New J. Phys. 11, 073051 (2009).

16. Monz, T. et al. 14-qubit entanglement: Creation and coherence. Phys. Rev. Lett. 106, 130506 (2011)

17. Shalm, L. K. et al. Three-photon energy-time entanglement. Nature Phys. 9, 19-22 (2012).

18. Aoki, T. et al. Experimental creation of a fully inseparable tripartite continuous-variable state. Phys. Rev. Lett. 91, 080404 (2003).

19. Armstrong, S. et al. Programmable multimode quantum networks. Nature Commun. 3, 1026 (2012).

20. Ansmann, M. et al. Violation of Bell's inequality in Josephson phase qubits. Nature 461, 504-506 (2009).

21. Werner, R. F. Quantum states with Einstein-Podolsky-Rosen correlations admitting a hidden-variable model. Phys. Rev. A 40, 4277-4281 (1989).

22. Handchen, V. et al. Observation of one-way Einstein-Podolsky-Rosen steering. Nature Photon. 6, 596-599 (2012).

23. He, Q. Y. \& Reid, M. D. Genuine multipartite Einstein-Podolsky-Rosen steering. Phys. Rev. Lett. 111, 250403 (2013).

24. Kimble, J. The quantum internet. Nature 453, 1023-1030 (2008).

25. Hillery, M., Buzek, V. \& Berthiaume, A. Quantum secret sharing. Phys. Rev. A 59, 1829-1834 (1999).
26. Branciard, C. et al. One-sided device-independent quantum key distribution: Security, feasibility, and the connection with steering. Phys. Rev. A 85, 010301(R) (2012).

27. Reid, M. D. Demonstration of the Einstein-Podolsky-Rosen paradox using nondegenerate parametric amplification. Phys. Rev. A 40, 913-923 (1989).

28. Bowen, W., Schnabel, R., Lam, P. K. \& Ralph, T. C. Experimental investigation of criteria for continuous variable entanglement. Phys. Rev. Lett. 90, 043601 (2003)

29. Palomaki, T. A., Teufel, J. D., Simmonds, R. W. \& Lehnert, K. W. Entangling mechanical motion with microwave fields. Science 342, 710-713 (2013).

30. Opanchuk, B., Arnaud, L. \& Reid, M. D. Detecting faked continuous-variable entanglement using one-sided device-independent entanglement witnesses. Phys. Rev. A 89, 062101 (2014)

\section{Acknowledgements}

This research was conducted by the Australian Research Council Centre of Excellence for Quantum Computation and Communication Technology (project number CE110001029) and has been supported by the Australian Research Council DECRA and Discovery Project Grants schemes. S.A. is grateful for funding from the Australia-Asia Prime Minister's Award. R.Y.T. thanks Swinburne University for a Research SUPRA Award, and Q.H. thanks National Natural Science Foundation of China under Grant No. 11121091 and 11274025 . This work was supported in part by National Science Foundation Grant No. PHYS-1066293 and the hospitality of the Aspen Center for Physics.

\section{Author contributions}

S.A., P.K.L., Q.H. and M.D.R. conceived of and designed the experiment. S.A. and J.J. constructed and performed the experiment with supervision from H-A.B. and P.K.L. S.A., M.W., R.Y.T., Q.G., Q.H. and M.D.R. contributed equally to the theory. S.A designed and created the virtual networks, and conducted the data analysis. S.A., Q.H and M.D.R. wrote the manuscript, and all authors commented on drafts.

\section{Additional information}

Supplementary information is available in the online version of the paper. Reprints and permissions information is available online at www.nature.com/reprints. Correspondence and requests for materials should be addressed to S.A., Q.H. or M.D.R

\section{Competing financial interests}

The authors declare no competing financial interests. 